\title{
Study of electrochromic devices incorporating a polymer gel electrolyte component
}

\author{
A. Gonçalves ${ }^{1,3}$, G. Gonçalves ${ }^{1}$, E. Fortunato ${ }^{1, a}$, A. Marques ${ }^{1,3}$, A. \\ Pimentel $^{1}$, R. Martins ${ }^{1}$, M. Silva ${ }^{2}$, M. Smith ${ }^{2}$, J. Bela $^{3}$, J. Borges $^{3}$ \\ ${ }^{1}$ Materials Science Department/CENIMAT and CEMOP/UNINOVA, Campus da \\ Caparica, 2829-516 Caparica, Portugal \\ ${ }^{2}$ Department of Chemistry, University of Minho, 4710-057 Braga, Portugal \\ ${ }^{3}$ TEKELEC, Chão de Murches P.O. Box 1942750-903 Cascais, Portugal \\ aelvira.fortunato@fct.unl.pt
}

Keywords: Electrochromic materials, tungsten oxide, polymer gel electrolyte

\begin{abstract}
Electrochromic materials have attracted considerable attention during the last two decades as a consequence of their potential application in several different types of optical devices. Examples of these devices include intelligent windows and time labels. In this paper the authors describe results obtained with thin tungsten oxide films produced at room temperature by rf magnetron sputtering under an argon and oxygen atmosphere on transparent conductive oxide coated glass substrates. To protect the surface of the electrochromic film, prevent water absorption and obtain a good memory effect under open circuit voltages, a layer of $\mathrm{Ta}_{2} \mathrm{O}_{5}$ was deposited over the $\mathrm{WO}_{3}$ films.

In this study, the effect of different electrolyte compositions on the open circuit memory of optical devices has been characterized. The best results were obtained for electrochromic devices with polymer gel $\mathrm{p}(\mathrm{TMC})_{3} \mathrm{LiClO}_{4}$ and $\mathrm{p}(\mathrm{TMC})_{8} \mathrm{LiClO}_{4}$ electrolytes. These prototype devices present an overall transmittance of $\sim 75 \%$ in their bleached state and after coloration 40.5 and $52.5 \%$ respectively. These devices also show memory effect and an optical density considered satisfactory for some electrochromic applications.
\end{abstract}

